\title{
Inventory of environmental certifications throughout the world
}

\author{
V. Lempereur, M. Balazard et C. Herbin \\ Institut Français de la Vigne et du Vin, France
}

\begin{abstract}
During the XXth century, intensive agricultural practices has been spread and allowed an important increase of productivity, especially thanks to scientific and technical innovations. Nevertheless, this change had consequences on biodiversity, quality of ecosystems and landscapes by a simplification of wine areas. In addition, sociocultural pressures toward agricultural practices had become stronger and stronger. In response, many mobilizations had begun to emerge, especially in the wine sector. For some years, we observed a real dynamic of the sector at the environmental level with the creation of many approaches which are promoting sustainable wine practices. These certifications are various and come up to consumers' wishes for products which are respectful of environment. They can be carried by productors associations, wine regions, cooperatives, wine companies or public authorities. We have inventoried in 22 countries a total of 54 environmental certifications among which 33 are dedicated to viticulture. We categorize them through: the scale (local, national, international), the area (vine, vine and wine, multi agricultural sector), the subjects treated (environment, economic, social) and the dynamic of the approach (voluntary, compulsory, individual or collective). This article informs and sensitizes the actors of wine sector to the diversity of environmental approaches in the world.
\end{abstract}

\section{Introduction}

Les pratiques d'agriculture intensive, qui se sont répandues au XXe siècle, ont permis une importante augmentation de la productivité, notamment grâce à de nombreuses innovations scientifiques et techniques. Néanmoins, le changement des pratiques a eu des conséquences, notamment sur la biodiversité, la qualité des écosystèmes et les paysages par une simplification des espaces viticoles. En plus de ces conséquences, les pressions socioculturelles vis-à-vis des pratiques agricoles sont devenues de plus en plus fortes. En réponse, de nombreuses mobilisations ont commencé à émerger, en particulier au niveau de la filière viticole.

Depuis la fin des années 1990, on peut observer une réelle dynamique de la filière au niveau environnemental avec la création de nombreuses certifications promouvant des pratiques viticoles durables. Ces démarches sont très diverses et viennent répondre, en partie, à une demande très forte des consommateurs pour des produits respectueux de l'environnement. Les démarches de certification rencontrent un réel intérêt auprès de la profession viticole. Elles peuvent être portées par des groupements de producteurs, des régions viticoles, des coopératives, des maisons de vin ou encore des pouvoirs publics.

La présence de label sur les bouteilles permet notamment de toucher certains marchés et de pouvoir se tourner plus facilement vers l'export. La présence de label sur les bouteilles de vin informe les consommateurs d'actions vis à vis du développement durables réalisées par les producteurs.

Chaque démarche environnementale dispose de son cadre propre, son propre domaine d'action, avec un périmètre spécifique. Les actions effectivement réalisées sont évaluées par l'intermédiaire d'un audit. L'audit est pratiqué par un organisme interne ou un organisme certificateur externe. L'attribution d'un logo est conditionnée aux résultats de l'audit. En France, suite au Grenelle de l'environnement (2007), les certifications environnementales des exploitations agricoles sont encadrées par le ministère de l'Agriculture. La mention « issue d'une exploitation de haute valeur environnementale » est une mention valorisante, tout comme la dénomination européenne « produit de montagne » et « produit de la ferme $\gg[1]$.

Aujourd'hui, il existe également au niveau européen quatre signes de qualité : l'appellation d'origine protégée (AOP), l'indication géographique protégée (IGP), la spécialité traditionnelle garantie (STG) et l'Agriculture Biologique $(\mathrm{AB})$. Les signes de qualité sont définis par le règlement européen (UE) $\mathrm{n}^{\circ} 1308 / 2013$ et se distinguent des mentions valorisantes [2].

\section{Méthodologie}

En 2017, l'Institut français de la vigne et du vin (IFV), en collaboration avec l'INAO, a publié un Guide de l'agroécologie en viticulture [3]. Le guide a pour objet de fournir des points de repère techniques pour tous les vignerons qui souhaitent, sur le plan individuel ou dans un cadre collectif, faire évoluer leurs pratiques, afin d'initier ou d'approfondir une démarche agroenvironnementale. Ce guide présente les leviers d'actions agroécologiques en viticulture organisés selon les cinq thématiques suivantes :

- Préservation et développement de la biodiversité,

- Maîtrise/réduction de la fertilisation, 
- Diminution de l'usage de produits phytosanitaires \& développement du biocontrôle,

- Recherche d'une meilleure gestion de l'eau,

- Recours à un matériel végétal plus adapté.

En 2018, l'IFV a entrepris la révision du guide de l'agroécologie en y intégrant une portée internationale, L'objectif est de produite pour 2020 le Guide de l'agroécologie en viticulture à l'international, en anglais et en français. Le travail d'inventaire des démarches de certification environnementale dans les différents pays producteurs de vin s'inscrit dans ce contexte.

\subsection{Recherche de l'information}

Le premier travail a consisté à identifier tous les pays producteurs de vins dans le monde. Pour cela, deux sources d'informations ont été croisées : les statistiques de la vitiviniculture dans le monde en 2017 produits par l'Organisation Internationale de la Vigne et du Vin, et les statistiques mondiaux de la production de vin en 2017 réalisés par The Wine Institute.

Pour chaque pays producteur de vin, un travail de recensement des démarches environnementales a été entrepris à partir d'informations trouvées sur internet et via une recherche active auprès de contacts de la filière viticole dans différents pays.

Après plusieurs mois de recherches, nous proposons de présenter les résultats obtenus. Néanmoins, ce travail ne prétend pas être exhaustif sur les certifications environnementales existantes.

Ce travail de recensement comporte certaines limites. Nous avons notamment rencontré des difficultés concernant l'accès à l'information : l'étendue, l'ancienneté, l'origine, les champs d'actions, les documents de référence, le champ de certification, le nombre d'exploitations certifiés et les volumes correspondants, etc. Du fait d'un manque de données pour certains points, nous avons été contraints de nous focaliser que sur un nombre de champ d'information restreint.

\subsection{Catégorisation}

A travers ce recensement international, nous avons pu constater une très grande diversité de certifications environnementales. Chacune a des caractéristiques bien précises concernant :

- l'origine (voir paragraphe 3.1) ;

- l'étendue (locale, régionale, nationale ou internationale) ;

- le champ de certification (toute l'exploitation, le raisin, le vin, le vignoble, la cave, le processus) ;

- les filières agricoles pouvant y avoir accès (vins, multifilières) ;

- les thématiques majeures (environnement, social, économique) et les champs d'actions principaux.

\section{Résultats}

Un total de 66 pays producteurs de vin dans le monde a été comptabilisé. Nous avons recensé au total 54 certifications environnementales, dont 33 uniquement dédiées à la viticulture, dans 22 pays différents.

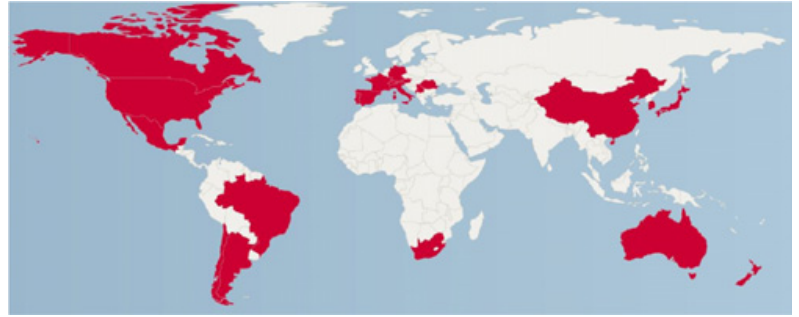

Figure 1. Localisation des pays dans lesquels des certifications environnementales ont été recensées.

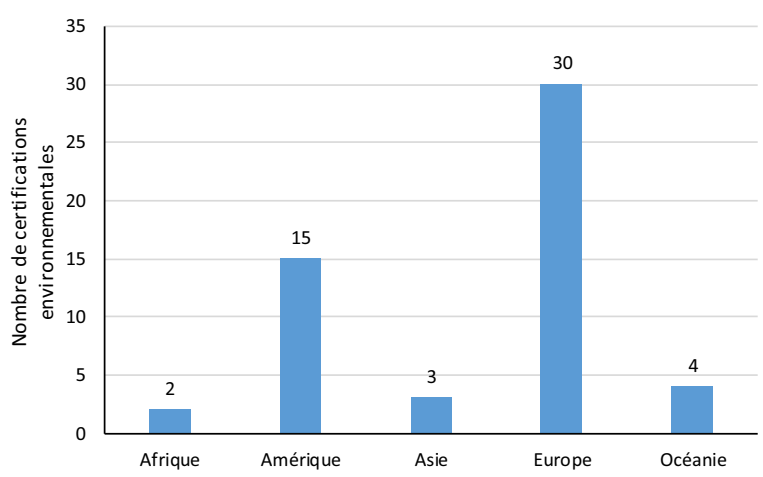

Figure 2. Nombre de certifications environnementales par continent.

\subsection{Le recensement des certifications}

Après étude de chaque certification, nous avons réalisé une présentation sous forme de tableaux (ci-dessous). Cet inventaire ne peut être exhaustif, du fait du grand nombre de certifications existantes. Pour plus de clarté, nous avons présenté les certifications par continent.

Nous avons également fait le choix de consacrer un point aux certifications d'agriculture biologique et à la biodynamie (voir paragraphe 3.4) ainsi qu'aux normes internationales (voir paragraphe 3.5). Certaines certifications seront donc abordées au cours de l'article sans pour autant apparaître au sein des tableaux d'inventaire.

En Afrique, des vignes sont présentes dans différents pays, au nord comme au sud du continent. Bien que nous avons pu recenser plusieurs approches en faveur de l'environnement en viticulture, nous n'avons pu répertorier des certifications environnementales qu'en Afrique du Sud.

Pour les certifications environnementales viticoles recensées en Amérique du Nord, et plus précisément aux Etats-Unis et au Canada, nous avons pu noter qu'elles ont exclusivement une étendue régionale. Chaque démarche s'applique sur un bassin de production viticole bien précis. Nous n'avons pu répertorier une certification nationale, exceptée pour USDA Organic (certification bio) mais qui concerne toutes les filières agricoles.

Pour les deux certifications recensées en Amérique du Sud, elles ont pour particularité d'émaner d'un organisme ou regroupement national vitivinicole, Vinos de Chile au Chili et Bodegas de Argentina en Argentine.

Sur le continent européen, on retrouve des certifications environnementales dans les différents pays producteurs de vins. Les démarches sont très diverses : nationale ou régionale, principalement viticole mais 


\begin{tabular}{|c|c|c|}
\hline Pays & Certification & Champs d'actions \\
\hline $\begin{array}{c}\text { Afrique du Sud } \\
\text { (Etendue nationale) }\end{array}$ & 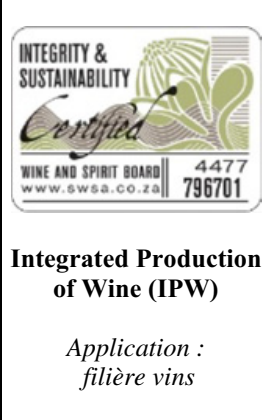 & $\begin{array}{c}\text { Environnement } \\
\text { Conduite de la vigne, } \\
\text { gestion des intrants } \\
\text { (fertilisation, irrigation, } \\
\text { traitements), traçabilité, } \\
\text { gestion durable de } \\
\text { l'énergie, réduction des } \\
\text { émissions carbone, } \\
\text { infrastructure et } \\
\text { équipement, processus de } \\
\text { vinification, gestion des } \\
\text { déchets solides, matières } \\
\text { sèches }\end{array}$ \\
\hline $\begin{array}{c}\text { Afrique du Sud } \\
\text { (Etendue nationale) }\end{array}$ & $\begin{array}{l}\text { CONSERVATION } \\
\text { CHAMPION } \\
\text { wwt.org.za }\end{array}$ & $\begin{array}{c}\text { Environnement } \\
\text { Réduction de l'empreinte } \\
\text { carbone, gestion durable } \\
\text { des ressources (eau, } \\
\text { énergie), protection des } \\
\text { écosystèmes (corridors et } \\
\text { habitats, plantes } \\
\text { invasives), gestion du } \\
\text { feu, gestion du bétail et } \\
\text { des animaux sauvages, } \\
\text { gestion des déchets }\end{array}$ \\
\hline
\end{tabular}

Figure 3. Certifications environnementales recensées en Afrique.

\begin{tabular}{|c|c|c|}
\hline Pays & Certification & Champs d'actions \\
\hline $\begin{array}{c}\text { Canada } \\
\text { (Etendue régionale : } \\
\text { Colombie- } \\
\text { Britannique) }\end{array}$ & $\begin{array}{c}\begin{array}{c}\text { Sustainable } \\
\text { Winegrowing } \\
\text { British Columbia }\end{array} \\
\text { Application: } \\
\text { filière vins }\end{array}$ & $\begin{array}{c}\text { Environnement, Social } \\
\text { gestion durable et } \\
\text { préservation des } \\
\text { ressources (écosystème, } \\
\text { eau, énergie), conduite } \\
\text { de la vigne, gestion } \\
\text { raisonnée de la et de la } \\
\text { protection du vignoble, } \\
\text { manutention des } \\
\text { matériaux, traitement } \\
\text { des déchets solides, } \\
\text { durabilité des relations } \\
\text { sociales }\end{array}$ \\
\hline
\end{tabular}

Figure 4. Certifications environnementales recensées en Amérique du Nord.

également multifilières pour les certifications biologiques, des champs de certification très variés (le vignoble, la cave, la structure, le processus), etc.

C'est le continent pour lequel nous avons pu recenser le plus grand nombre de certifications abouties mais également en cours d'élaboration.

Ayant réalisé un recensement exhaustif pour les certifications environnementales en France, nous avons jugé opportun de présenter ces démarches à part du tableau consacré au continent européen. En plus des démarches présentées ci-dessus, il existe également les labels $\mathrm{AB}$ (Agriculture Biologique) et Biodyvin (biodynamie), mais celles-ci seront abordées dans le paragraphe 3.4. En France, les viticulteurs ont accès à des certifications nationales mais également régionales. Chaque bassin

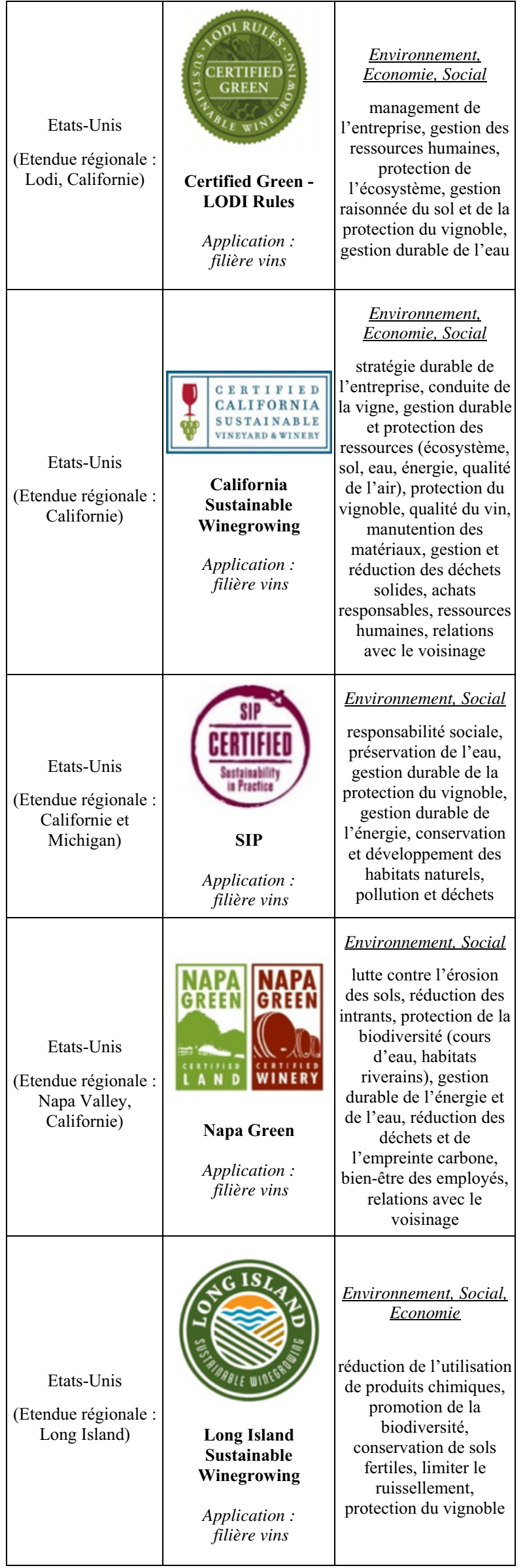

Figure 4. Continued. 


\begin{tabular}{|c|c|c|}
\hline $\begin{array}{c}\text { Etats-Unis } \\
\text { (Etendue régionale : } \\
\text { Oregon, Washington } \\
\text { et Idaho) }\end{array}$ & $\begin{array}{l}\text { LIVE IVE } \\
\text { CERTIFIED CERTIFIED } \\
\text { SUSTAANABLE SUINABLE } \\
\text { GRAPES WINE } \\
\text { LIVE Certified } \\
\text { Sustainable } \\
\text { Application: } \\
\text { filière vins }\end{array}$ & $\begin{array}{c}\text { Environnement, Social } \\
\text { gestion durable des } \\
\text { ressources (biodiversité, } \\
\text { sol, eau, énergie), aucun } \\
\text { OGM autorisé, } \\
\text { protection du vignoble } \\
\text { réduction des } \\
\text { herbicides, réduction } \\
\text { des émissions des gaz à } \\
\text { effet de serre, gestion } \\
\text { du matériel, bien-être et } \\
\text { sécurité des employés, } \\
\text { impacts sur la } \\
\text { communauté et } \\
\text { éducation du public }\end{array}$ \\
\hline
\end{tabular}

Figure 4. Continued.

\begin{tabular}{|c|c|c|}
\hline Pays & Certification & Champs d'actions \\
\hline $\begin{array}{c}\text { Argentine } \\
\text { (Etendue nationale) }\end{array}$ & $\begin{array}{c}\text { SUSTENTABILIDAD } \\
\text { CER R IFICADA } \\
\text { Certificado de } \\
\text { sustentabilidad - } \\
\text { Bodegas de Argentina } \\
\text { Application: } \\
\text { filière vins }\end{array}$ & $\begin{array}{c}\text { Environnement, social } \\
\text { gestion durable des } \\
\text { ressources (sol, eau, } \\
\text { énergie, qualité de l'air) } \\
\text { protection du vignoble, } \\
\text { protection de } \\
\text { l'écosystème, gestion et } \\
\text { utilisation des matériaux, } \\
\text { réduction et gestion des } \\
\text { résidus solides, achats } \\
\text { responsables, ressources } \\
\text { humaines, voisinage et } \\
\text { communauté }\end{array}$ \\
\hline $\begin{array}{c}\text { Chili } \\
\text { (Etendue nationale) }\end{array}$ & \begin{tabular}{|c|} 
Código de \\
Sustentabilidad - Vinos \\
de Chile \\
Application: \\
filière vins
\end{tabular} & $\begin{array}{c}\frac{\text { Environnement, Social, }}{\text { Economie }} \\
\text { Conduite du vignoble, } \\
\text { réduction des déchets et } \\
\text { recyclage, gestion } \\
\text { durable des ressources } \\
\text { (énergie, eau), sécurité } \\
\text { au travail, prévention } \\
\text { contre toute } \\
\text { contamination, politique } \\
\text { de durabilité, éthique, } \\
\text { relations humaines, } \\
\text { droits humains, } \\
\text { changement climatique, } \\
\text { qualité de vie au travail, } \\
\text { communauté, } \\
\text { commercialisation, } \\
\text { durabilité économique }\end{array}$ \\
\hline
\end{tabular}

Figure 5. Certifications environnementales recensées en Amérique du Sud.

viticole tend à structurer sa propre démarche afin d'inciter les viticulteurs à adapter des pratiques plus respectueuses de l'environnement.

Pour les certifications environnementales viticoles recensées en Océanie, elles ont pour particularité d'être toutes applicables au niveau national, même si cela n'a pas toujours été le cas pour Sustainable Australia Winegrowing. Concernant leur filière d'application, elles sont toutes dédiées à la viticulture, même si la démarche Freshcare a également été déclinée pour d'autres filières.

\begin{tabular}{|c|c|c|}
\hline Pays & Certification & Champs d'actions \\
\hline $\begin{array}{c}\text { Autriche } \\
\text { (Etendue nationale) }\end{array}$ & $\begin{array}{c}\text { NACHHALTIG } \\
\text { AUSTRIA } \\
\text { NACHHALTIG } \\
\text { AUSTRIA.AT } \\
\text { ZERTIFIIERT 1234567 } \\
\\
\text { Nachhaltig Austria - } \\
\text { Zertifiziert 1234567 } \\
\text { Application: } \\
\text { filière vins }\end{array}$ & \begin{tabular}{|c}
$\frac{\text { Environnement, Social, }}{\text { Economie }}$ \\
conduite de la vigne, \\
protection du vignoble et \\
matériel utilisé, gestion \\
durable du sol (travaux, \\
fertilisation, \\
enherbement), protection \\
de la biodiversité et des \\
éléments paysagers, \\
utilisation des machines, \\
gestion durable de \\
l'énergie et de l'eau, \\
vinification, matières \\
sèches, sécurité au \\
travail, économie durable \\
et locale
\end{tabular} \\
\hline $\begin{array}{c}\text { Espagne } \\
\text { (Etendue régionale : } \\
\text { Costers del Segre) }\end{array}$ & $\begin{array}{c}\text { Programa de } \\
\text { sostenibilitat do } \\
\text { costers del Segre } \\
\text { Application: } \\
\text { filière vins }\end{array}$ & $\begin{array}{c}\text { Environnemental, Social, } \\
\text { Economique } \\
\text { conduite de la vigne, } \\
\text { protection du vignoble, } \\
\text { gestion du sol, irrigation, } \\
\text { préservation de } \\
\text { l'environnement et } \\
\text { paysage, traitement des } \\
\text { déchets, sécurité et } \\
\text { hygiène, cohésion } \\
\text { sociale, oenotourisme }\end{array}$ \\
\hline $\begin{array}{c}\text { Espagne } \\
\text { (Etendue nationale) }\end{array}$ & $\begin{array}{l}\text { For Climate } \\
\text { Protection Wineries } \\
\text { Application : } \\
\text { filière vins }\end{array}$ & $\begin{array}{l}\text { Environnement } \\
\\
\text { réduction des gaz à effet } \\
\text { de serre, gestion durable } \\
\text { de l'eau, réduction des } \\
\text { résidus, gestion } \\
\text { énergétique et énergies } \\
\text { renouvelables }\end{array}$ \\
\hline $\begin{array}{c}\text { Italie } \\
\text { (Etendue régionale : } \\
\text { Sicile) }\end{array}$ & $\begin{array}{l}\text { SOStain } \\
\text { Application: } \\
\text { filière vins }\end{array}$ & $\begin{array}{l}\text { Environnement } \\
\text { mesurer l'impact des } \\
\text { traitement (sur l'air, } \\
\text { l'eau, le vignoble, le } \\
\text { territoire), interdiction de } \\
\text { désherbage chimique, } \\
\text { utilisation minimale de } \\
\text { l'énergie, vinification } \\
\text { (sulfites), encrage local, } \\
\text { protection de la } \\
\text { biodiversité, utilisation } \\
\text { de matériaux recyclables } \\
\text { et biodégradables, } \\
\text { transparences des actions } \\
\text { et résultats }\end{array}$ \\
\hline
\end{tabular}

Figure 6. Certifications environnementales recensées en Europe.

\subsection{Les certifications environnementales : diversité des origines}

La première caractéristique d'une certification est son origine. Le recensement international a permis de 


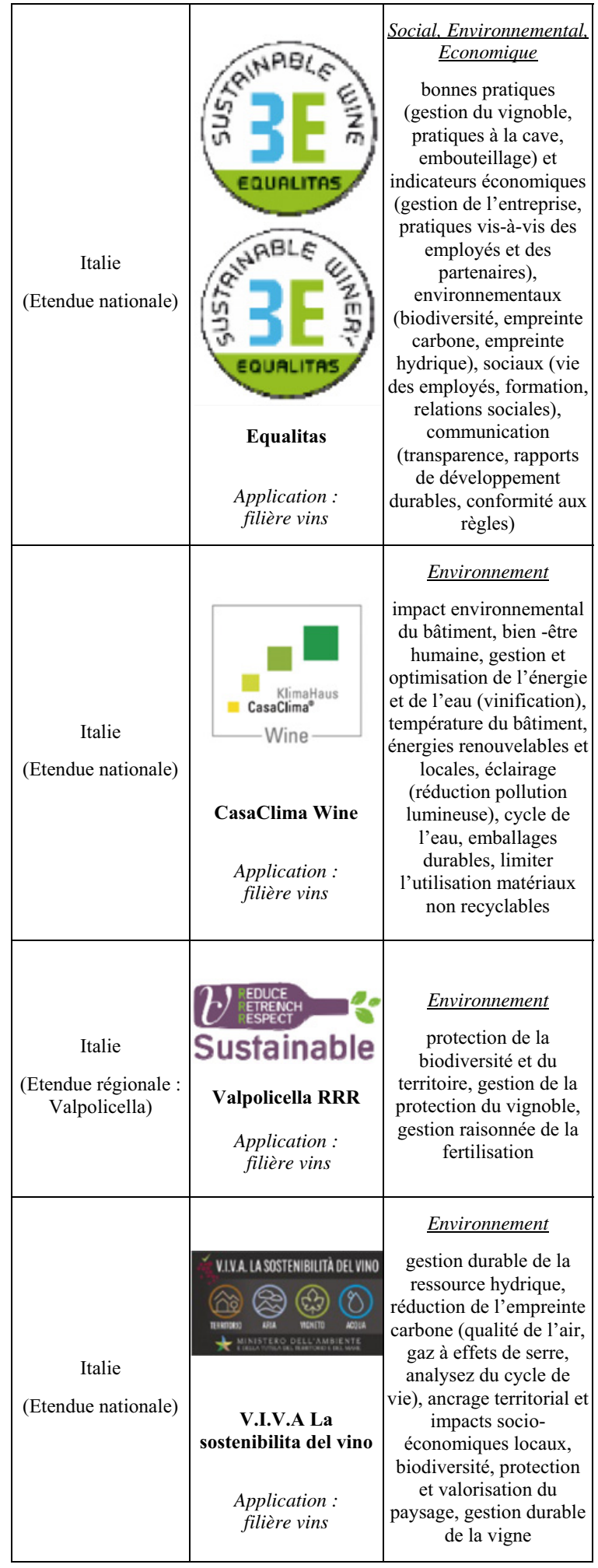

Figure 6. Continued.

montrer une multitude d'impulsions différentes. Ainsi, une certification environnementale peut émaner de :

- L'Etat, comme par exemple la certification Haute Valeur Environnementale qui a été élaborée par le ministère de l'Agriculture (France) ;

- d'un programme national, tel que la démarche V.I.V.A (Italie) qui vient faire suite au programme VIVA Sustainable Wine développé par le ministère de l'Environnement, de la protection du territoire et de la

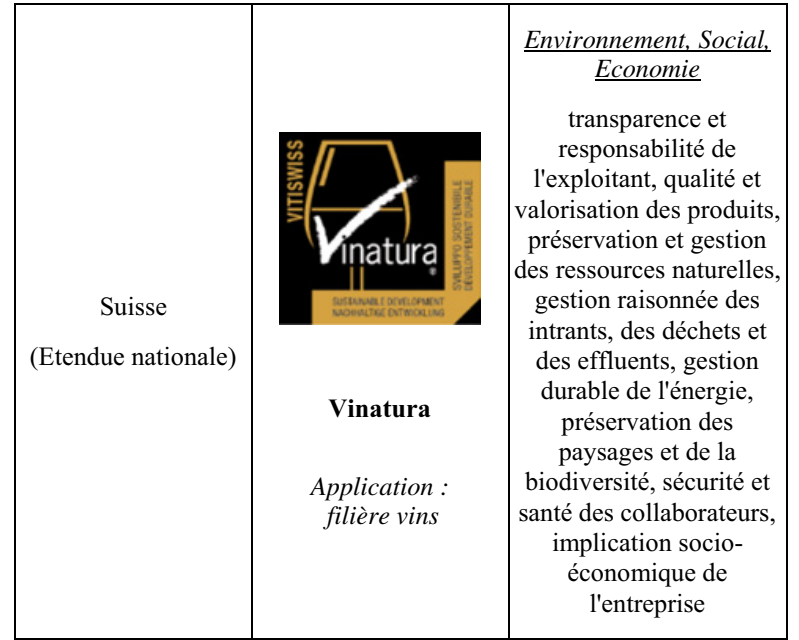

Figure 6. Continued.

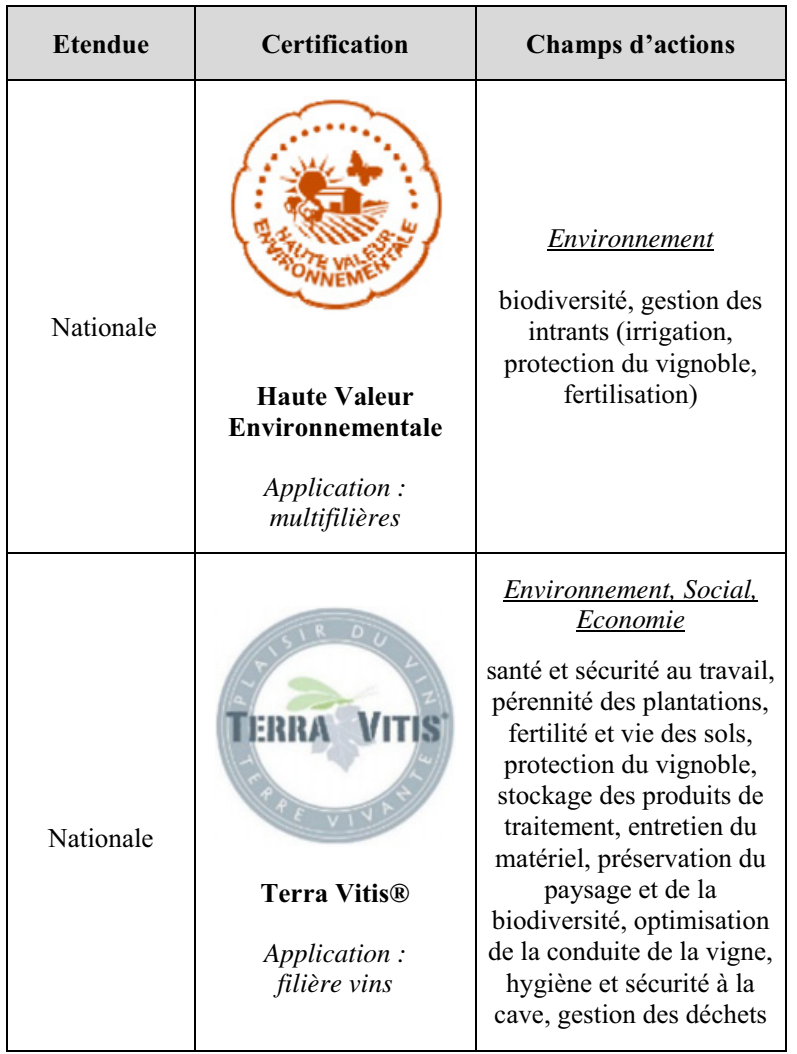

Figure 7. Certifications environnementales recensées en France.

mer, et mis en œuvre par le centre de recherche pour le développement durable en agriculture (OPERA) ;

- un organisme national de la filière vins, comme la Österreichische Weinbauverband (l'Association autrichienne de la viticulture) qui a développé la démarche Nachhaltig Austria ou encore l'industrie viticole d'Afrique du Sud qui a élaboré le programme Integrated Production of Wine (IPW) ;

- une ONG, comme WWF qui a mis au point la certification Conservation Champion visant à préserver et développer la biodiversité au sein des vignobles (Afrique du Sud) ;

- un rassemblement de différentes structures, par exemple la synergie entre des leaders de l'industrie 


\begin{tabular}{|c|c|c|}
\hline Nationale & $\begin{array}{l}\text { Vignerons en } \\
\text { Développement } \\
\text { Durable } \\
\text { Application: } \\
\text { filière vins }\end{array}$ & \begin{tabular}{|}
$\frac{\text { Environnement, Social, }}{\text { Economie }}$ \\
gouvernance et pratiques \\
managériales, performance \\
économique, responsabilité \\
sociale (équité, intégration \\
territoriale, santé, sécurité), \\
responsabilité \\
environnementale (gestion \\
de l'eau, consommation \\
énergétique, pollution de \\
l'air, traitement des \\
déchets, gestion des sols, \\
biodiversité, éco- \\
conception, transports)
\end{tabular} \\
\hline $\begin{array}{l}\text { Régionale : } \\
\text { Champagne }\end{array}$ & $\begin{array}{c}\text { Viticulture durable en } \\
\text { champagne } \\
\text { Application: } \\
\text { filière vins }\end{array}$ & \begin{tabular}{|c} 
Environnement \\
gestion de l'exploitation, \\
aménagement et \\
établissement du vignoble \\
et des structures \\
d'exploitation, nutrition du \\
sol et de la vigne, entretien \\
des sols, conduite de la \\
vigne, protection raisonnée \\
du vignoble, mise en \\
œuvre de protection de la \\
vigne, gestion des déchets
\end{tabular} \\
\hline $\begin{array}{l}\text { Régionale : } \\
\text { Cognac }\end{array}$ & $\begin{array}{l}\text { Viticulture durable } \\
\text { Cognac } \\
\text { Application: } \\
\text { filière vins }\end{array}$ & $\begin{array}{l}\text { Environnement, Social } \\
\text { pérennité du vignoble, } \\
\text { protection du milieu } \\
\text { naturel, maitrise des } \\
\text { produits phytosanitaires, } \\
\text { gestion des effluents de } \\
\text { chais et de distillerie, } \\
\text { formation, santé, sécurité, } \\
\text { viticulture et voisinage }\end{array}$ \\
\hline $\begin{array}{l}\text { Régionale : } \\
\text { Bordeaux }\end{array}$ & $\begin{array}{c}\text { Système de } \\
\text { Management } \\
\text { environnemental du } \\
\text { vin de Bordeaux } \\
\text { Application: } \\
\text { filiere vins }\end{array}$ & $\begin{array}{l}\text { Environnement, Social } \\
\text { respect du référentiel de la } \\
\text { norme ISO 14001, } \\
\text { préservation de la } \\
\text { biodiversité et de la } \\
\text { ressource en eau, gestion } \\
\text { durable de l'énergie, } \\
\text { gestion des déchets, } \\
\text { limitation du recours aux } \\
\text { intrants, environnement de } \\
\text { vie et de travail, } \\
\text { participation active des } \\
\text { collaborateurs, gestion } \\
\text { durable de l'entreprise, } \\
\text { innovation, diffusion et } \\
\text { partage d'expérience }\end{array}$ \\
\hline
\end{tabular}

Figure 7. Continued.

viticole, de groupes environnementaux et d'agences gouvernementales qui a été à l'origine de la certification Napa Green Land (Etats-Unis) ;

- la philosophie d'un homme, tel que Karl Schefer qui, de par sa conviction que l'agriculture biologique ne peut avoir lieu sans une harmonie entre l'homme et la nature, a mis au point la démarche DELINAT (Suisse);

\begin{tabular}{|c|c|c|}
\hline Pays & Certification & Champs d'actions \\
\hline $\begin{array}{c}\text { Australie } \\
\text { (Etendue nationale) }\end{array}$ & $\begin{array}{l}\text { Uistailnable } \\
\text { AUSTRALA winegrowing } \\
\text { Sustainable Australia } \\
\text { Winegrowing } \\
\text { Application: } \\
\text { filiere vins }\end{array}$ & $\begin{array}{c}\text { Environnement, } \\
\text { Economie, Social } \\
\text { santé du sol et gestion } \\
\text { de la fertilisation, } \\
\text { protection du } \\
\text { vignoble, protection } \\
\text { de la biodiversité, } \\
\text { gestion durable de } \\
\text { l'eau et des déchets, } \\
\text { relations sociales, } \\
\text { économie durable }\end{array}$ \\
\hline $\begin{array}{c}\text { Australie } \\
\text { (Etendue nationale) }\end{array}$ & $\begin{array}{c}\text { Freshcare } \\
\text { Freshcare environmental } \\
\text { viticulture } \\
\text { Application: } \\
\text { filierre vins }\end{array}$ & $\begin{array}{c}\text { Environnement } \\
\text { gestion et protection } \\
\text { du sol (érosion, } \\
\text { contamination, } \\
\text { fertilisation), gestion } \\
\text { de la protection du } \\
\text { vignoble (minimiser } \\
\text { les risques pour } \\
\text { l'environnement), } \\
\text { protection de la } \\
\text { biodiversité, gestion } \\
\text { des déchets, qualité } \\
\text { de l'air, gestion } \\
\text { durable de l'énergie } \\
\text { et de l'eau, stockage } \\
\text { du fioul }\end{array}$ \\
\hline $\begin{array}{l}\text { Nouvelle-Zélande } \\
\text { (Etendue nationale) }\end{array}$ & $\begin{array}{l}\text { O SUSTAINABLE } \\
\text { WINEGROWING } \\
\text { Sustainable Winegrowing } \\
\text { Application: } \\
\text { filierre vins }\end{array}$ & $\begin{array}{l}\text { Environnement. } \\
\text { Social, Economie } \\
\text { préservation de la } \\
\text { biodiversité, gestion } \\
\text { et protection du sol } \\
\text { (fertilisants et } \\
\text { nutriments, } \\
\text { enherbement, } \\
\text { qualité), gestion } \\
\text { durable de l'eau et de } \\
\text { l'énergie, qualité de } \\
\text { l'air, protection } \\
\text { durable du vignoble, } \\
\text { gestion des déchets et } \\
\text { recyclage, bien-être } \\
\text { des employés, } \\
\text { durabilité } \\
\text { économique }\end{array}$ \\
\hline
\end{tabular}

Figure 8. Certifications environnementales recensées en Océanie.

- un organisme certificateur, comme Bureau Veritas (France) qui a développé, en partenariat avec la société Dervenn et des experts, la certification Biodiversity Progress $^{\oplus}$, accessible à tout type d'entreprise afin d'améliorer les actions en faveur de la biodiversité et la préservation des ressources naturelles et des services éco-systémiques. Cette certification peut notamment être utile aux entreprises désirant s'engager dans la Responsabilité Sociétale des Entreprises, car elle reprend les différentes obligations environnementales de l'ISO 26000 ;

- une volonté de protéger un milieu naturel, tel que la certification Fish Friendly Farming qui a été créée en vue de protéger la rivière Russian (EtatsUnis, Californie). Les exploitations longeant la rivière, majoritairement viticoles, peuvent s'engager dans la démarche afin de veiller à la conservation du sol et 
de l'eau, la gestion et la restauration des ruisseaux et des rivières riveraines, tout en limitant l'utilisation d'intrants ;

- une commission vitivinicole comme celle de la région d'Alentejo (Portugal) qui a décidé depuis plusieurs années d'aller vers une certification environnementale : le Plano de Sustentabilidade dos Vinhos do Alentejo. Ce plan prend exemple sur des certifications existantes, notamment en Californie. Le programme a été divisé en différents niveaux pour sensibiliser plus facilement les viticulteurs et leur permettre d'avoir une meilleure appropriation de la démarche. Chaque viticulteur peut donc se positionner à travers plusieurs niveaux, d'une première approche de la pratique jusqu'au système de management complet. Ce projet a été réalisé à partir : de scénarios portant sur des thématiques communes à tous (maladies, ressources humaines, etc.), de workshops où les producteurs ont pu s'exprimer sur leurs attentes et priorités d'actions, de partenariats avec les universités pour identifier les points importants sur lesquels travailler. Ce programme, débuté en 2015, regroupe plus d'une grosse centaine de membres. Pour l'instant, la démarche est volontaire et ne dispose pas d'un logo apposable sur les produits commercialisés.

Pour résumer, il existe autant d'origines différentes de certifications que de certifications.

\subsection{Les outils à dispositions des viticulteurs}

Pour les viticulteurs désirant s'engager au sein d'une démarche environnementale, ils ont possibilité d'accéder à des documents pouvant les aider dans leurs réflexions. En effet, de nombreuses certifications mettent à disposition gratuitement des documents d'appui, des listes détaillées d'actions évaluées dans le cadre de la démarche, des tableaux pour réaliser un autodiagnostic avec différents paliers d'application en fonction des actions, des exemples de programmes à mettre en place, des documents de référence (études, recherches scientifiques, exemples de cas concret, etc.).

Mais certaines démarches ont développé des outils encore plus complets. C'est le cas de la certification Nachhaltig Austria (Autriche) qui met à disposition gratuitement un logiciel en ligne d'auto-évaluation. Les viticulteurs peuvent donc accéder à cet outil.

Après une inscription rapide mais anonyme, il est possible d'accéder à une grille d'évaluation qui comporte cinq volets : la production des raisins, la production de vin, la plantation du vignoble, les affaires sociales, l'économie. En tout, l'outil permet d'évaluer pas loin de 340 activités de l'exploitation. Les activités évaluées tiennent compte de la localisation du vignoble (renseignée lors de la phase d'inscription gratuite). Pour chaque activité renseignée, des informations sont données concernant son impact sur un ensemble de thématiques (sol, biodiversité, consommation de matière, eau, climat, énergie, qualité, social, économie). A la fin de l'autodiagnostic, l'outil génère un diagramme en toile d'araignée qui permet de situer les activités de l'exploitation en fonction des neuf thématiques.

Avec l'aide de ce logiciel, les viticulteurs peuvent donc se situer et travailler sur les activités qui n'ont pas encore atteint le niveau maximal de durabilité.
L'outil a été conçu afin de permettre aux viticulteurs d'avoir un développement continu dans leurs actions, l'autodiagnostic pouvant être effectué autant de fois que souhaité et de façon régulière. Si les objectifs de durabilité sont atteints, le viticulteur peut, s'il le souhaite, demander la certification Nachhaltig Austria.

\subsection{L'Agriculture Biologique et la Biodynamie}

L'agriculture biologique, et tout particulièrement la production de vin bio, est régie par des pratiques bien précises comme : l'interdiction d'utiliser des produits chimiques de synthèse et des OGM, de limiter l'emploi d'intrants, de suivre un processus de vinification règlementé (additifs et auxiliaires œnologiques autorisés, teneur en $\mathrm{SO}_{2}$, etc.).

Aujourd'hui, les certifications bio sont omniprésentes sur les marchés mondiaux. A ce jour, on peut recenser un grand nombre de certifications nationales : Australian Certified Organic (Australie), Organico Brasil (Brésil), Canada Organic (Canada), China National Organic Product Standard GBT19630 (Chine), KOR (Corée du Sud), JAS (Japon), Organico SAGARPA Mexico (Mexique), KRAV (Suède), Biosuisse (Suisse), USDA Organic (Etats-Unis), etc.

En Europe, on retrouve ce système de certification d'agriculture biologique au niveau national (logo AB en France, Agricultura Ecologica en Roumanie, etc.) mais également au niveau communautaire. En effet, l'Union Européenne (UE) a également élaboré sa propre certification, connu sous le visuel de l'Eurofeuille. Ce label est obligatoirement à apposer sur tout produit issu de l'agriculture biologique au sein des pays de l'UE. L'avantage de cette démarche est qu'elle est reconnue par un groupe de pays, ce qui facilite la circulation des produits.

L'Eurofeuille n'est cependant pas le seul label bio qui se retrouve dans plusieurs pays. On peut notamment citer la certification Naturland, initialement impulsée en Allemagne et qui se retrouve actuellement dans une cinquantaine de pays dans le monde.

Au sein des certifications biologiques, on a également pu noter quelques spécificités suivant les démarches. Pour la viticulture biologique, il existe par exemple la certification Delinat. Initialement impulsée en Suisse, elle s'est aujourd'hui étendue à plusieurs pays européens. Cette démarche accorde une grande importance à la biodiversité. Ainsi, dans le cahier des charges, il est demandé, entre autres, d'enherber les parcelles, de minimiser le travail du sol, de réaliser des compensations écologiques ou encore de privilégier une régulation naturelle (interdiction des produits chimiques de synthèse).

En Allemagne, on trouve la certification ECOVIN, qui s'applique uniquement à la filière vins. Cette démarche vient attester des pratiques au vignoble, mais également à la cave, qui sont en conformité avec la production d'un vin biologique.

Toujours en Allemagne, on a pu recenser la certification Bioland. Applicable à toutes les filières agricoles, cette démarche n'atteste pas seulement du processus de réalisation du produit biologique mais également des pratiques en faveur du développement local (social et économique). 


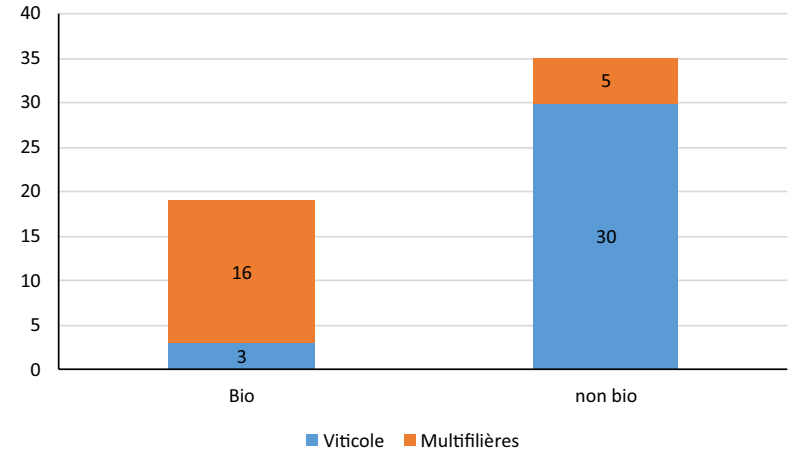

Figure 9. Nombre de certifications environnementales biologiques, viticoles ou multifilières.

Pour résumer, sur toutes les certifications biologiques recensées (19 au total), seules 3 sont spécifiquement dédiées à la viticulture. Les certifications biologiques sont donc majoritairement multifilières, même s'il y a certaines particularités propres à quelques filières. Au contraire, pour les certifications non biologiques (35), nous avons recensé une très forte majorité de démarches dédiées uniquement à la filière vins (30) (cf Fig. 9).

Il existe également des certifications dédiées à la biodynamie. Cette démarche a été impulsée par Rudolf Steiner, agronome autrichien, et s'est concrétisée par une certification : Demeter. Initialement mise en oeuvre en Allemagne, Demeter est aujourd'hui en application dans une soixantaine de pays. Les principes de la biodynamie sont entre autres : la protection du vignoble à partir de matières végétales, animales et minérales transformées, ainsi que l'importance des rythmes terrestres et lunaires dans la conduite de la vigne.

Cette démarche a également été transposée à échelle nationale. Par exemple, en France on trouve la certification Biodyvin, axée sur la biodynamie et spécifiquement dédiée à la filière vins. La particularité de cette certification est que, pour les viticulteurs désirant être labellisés Biodyvin, ils doivent au préalable être certifiés en agriculture biologique.

\subsection{Les normes internationales}

A plus grande échelle, il existe des normes environnementales internationales, accessibles aux entreprises vitivinicoles : ISO 14001 et ISO 26000.

Ces normes ont été conçues par l'Organisme International de la Normalisation. Elles ont été développées pour être applicables dans tous les pays et par tout type d'entreprise.

L'ISO 14001 fait partie d'un ensemble de normes ISO 14000, initialement créées afin d'impliquer les entreprises dans la sauvegarde de l'environnement. Elle repose sur le principe d'amélioration continue de la performance environnementale d'une entreprise. Cette norme définit les éléments caractéristiques d'un système de gestion efficace et écologique. Le but étant pour l'entreprise d'évaluer et de maîtriser les impacts de ses activités sur son environnement. En tout, la norme regroupe 18 exigences sur lesquelles l'entreprise doit chercher à s'améliorer.

L'ISO 26000, également connu sous la dénomination Responsabilité Sociétale des Entreprises (RSE), est définie comme «la responsabilité d'une entreprise vis-à-vis des impacts de ses décisions et de ses activités sur la société et sur l'environnement, se traduisant par un comportement transparent et éthique » [4]. L'entreprise doit élaborer un plan d'action annuel structuré autour des trois piliers du développement durable (environnement, social, économie).

En France, cette norme a été transposée au secteur de la vigne et du vin par un groupe de travail piloté par l'AFNOR. Il existe donc à présent un guide pour répondre aux enjeux du secteur et aider toute entreprise de la filière qui le désire à progresser dans la RSE [4].

Ces normes internationales sont souvent des dispositifs lourds et peu adaptés aux entreprises viticoles, notamment pour les petites structures. De plus, ces certifications ont un coût économique conséquent, ce qui peut être un frein pour de nombreuses petites entreprises viticoles. Toutefois, plusieurs initiatives tendent à rendre ces normes plus accessibles et plus compréhensibles pour la filière.

\section{Conclusion}

Ce travail d'inventaire international des certifications environnementales montre une grande diversité des démarches existantes, portant chacune sur des champs d'actions différents. L'objectif est de porter à connaissance et de sensibiliser les acteurs de la filière vitivinicole sur l'hétérogénéité des démarches environnementales dans le monde. Les initiateurs de ces démarches sont très divers et la propagation de la démarche peut aller, pour certaines, au-delà de la frontière du pays d'origine.

Il est toutefois regrettable que des données sur l'ampleur de la diffusion de chacune des certifications ne soient pas accessibles, comme le nombre d'exploitations certifiées, les surfaces concernées ou encore les volumes de vin produits sous ladite certification.

On peut également souligner l'initiative d'harmonisation à l'échelle internationale de normes environnementales telles que l'ISO 14001 et l'ISO 26000. Transposables dans différents secteurs d'activités, ces normes gagnent à être connues des consommateurs. Cependant, s'agissant d'une démarche de progrès portée par une entreprise, cet engagement ne peut donc être matérialisé par un logo sur une bouteille de vin. Cette absence de communication visuelle sur le produit final peut constituer un frein dans l'information auprès des consommateurs.

Une autre limite peut également être soulevée face aux si nombreuses initiatives menées afin de valoriser ses pratiques vertueuses auprès des consommateurs. Les études qualitatives exploratoires sur la perception des logos relatifs aux certifications environnementales montrent qu'à ce jour seuls les logos d'agriculture biologique sont connus et correctement compris des consommateurs [5]. Face à la multiplication des labels, il risque d'être difficile de communiquer de façon efficace auprès des consommateurs. Il serait donc à réfléchir, s'il ne serait pas possible de tendre vers une harmonisation des démarches. La filière vins disposant d'un organisme international, l'OIV, pourrait se servir de ce dernier pour essayer de standardiser les différentes approches existantes et ainsi essayer de rendre plus lisible les actions entreprises par la filière au niveau international. L'idée pourrait être 
de créer une certification environnementale internationale spécifique à la filière vins.

\section{Références}

[1] Code rural et de la pêche maritime, article R641-57-1 (2011)

[2] Règlement (UE) n 1308/2013 du 17 décembre 2013 portant organisation commune de marché des produits agricoles (produits viticoles), article 93 (2013)
[3] V. Lempereur, J. Gautier, C. Herbin, Bio Web Conf. 9 (2017)

[4] AFNOR, Accord AC X 30-032 - Développement durable et responsabilité sociétale, guide d'utilisation de la norme ISO 26000:2010 pour la filière vin (2015)

[5] E. Ginon, G. Ares, L.H.E. Dos Santos Laboissière, J. Brouard, S. Issanchou, R. Deliza, Food Res. Int. 62 (2014) 